\title{
Imaging of the spleen: what the clinician needs to know
}

\author{
Thomas Vancauwenberghe ${ }^{1}$, MD, Annemiek Snoeckx ${ }^{2}$, MD, Dirk Vanbeckevoort ${ }^{1}$, MD, Steven Dymarkowski ${ }^{1}$, MD, PhD, \\ Filip M Vanhoenacker ${ }^{2,3,4}$, MD, PhD
}

\begin{abstract}
The spleen is considered 'the forgotten organ' among radiologists and clinicians, although it is well visualised on abdominal computed tomography and magnetic resonance imaging. Moreover, the spleen is commonly involved in a wide range of pathologic disorders. These include congenital anomalies, infectious and inflammatory diseases, vascular disorders, benign and malignant tumours, and systemic disorders. In this review, we focus on the key imaging findings of the normal spleen, its variants, as well as relevant congenital and acquired abnormalities. It is of utmost importance to recognise and correctly interpret the variable spectrum of abnormalities that may involve the spleen, in order to avoid unnecessary invasive procedures and to guide adequate treatment.
\end{abstract}

Keywords: splenic infarction, splenic infection-inflammation, splenic tumours, splenic variants, splenomegaly

\section{INTRODUCTION}

Compared to imaging evaluation of other upper abdominal organs, requests for imaging of the spleen are relatively rare in daily clinical practice. However, the spleen may be involved in a large variety of congenital and acquired disorders. Significant overlap in imaging features among these various conditions is seen; therefore, clinical information is important. The purpose of this paper is to give a comprehensive overview of splenic imaging.

\section{NORMAL SPLEEN}

\section{Microscopic anatomy}

The splenic parenchyma consists of lymphatic follicles and reticuloendothelial cells, surrounding the arteries ('white pulp') and an interspersed network of vascular sinusoids ('red pulp'). ${ }^{(1)}$ The ratio of white to red pulp increases with age due to accumulated antigenic exposure and stimulation. There are two circulatory routes through the splenic pulp: open and closed circulation. The closed 'fast flow' circulation drains blood directly into the venous sinusoids, flowing together to become trabecular veins. ${ }^{(2)}$ In the open 'slow flow' circulation, blood flows into a reticular fibrous framework of the red pulp and/or via the marginal zone of the lymphatic tissue before reaching the sinusoids. ${ }^{(3)}$ These variable circulatory routes through the splenic pulp may explain the unique inhomogeneous early enhancement pattern of the spleen on computed tomography (CT) and magnetic resonance (MR) imaging. ${ }^{(4)}$

\section{IMAGING MODALITIES}

\section{Ultrasonography}

Ultrasonography (US) is frequently the first imaging modality used to evaluate the spleen (Fig. 1). On US, the spleen is homogeneous, slightly more echogenic than normal renal cortex, and iso- to slightly more hyperechoic than liver parenchyma. Colour Doppler is useful in the evaluation of vascular pathology in the splenic hilum. Splenic focal lesions are frequently subtle and often nonspecific, appearing as hypoechoic lesions. ${ }^{(5)}$ Therefore, every focal heterogeneity detected on US should be further evaluated by $\mathrm{CT}$ or MR imaging. (2)

\section{Computed tomography}

On unenhanced CT, the spleen is homogeneous with attenuation values ranging between 40 and 60 Hounsfield units (HU). Unenhanced images are mainly used for the detection of splenic calcifications. After intravenous contrast injection, the normal spleen enhances in a mottled pattern during the arterial and early portal venous phases (Fig. 2). Enhancement patterns are variable and include serpentine-cordlike, focal and diffuse heterogeneity. As mentioned previously, the enhancement pattern is caused by variable flow rates through the open and closed circulation. This inhomogeneous enhancement pattern should not be confused with splenic disease. Middle to late portal venous phases of contrast enhancement will show homogeneous enhancement of the normal spleen. Delayed phase scans 3 min after contrast injection are helpful for excluding splenic lacerations in posttraumatic patients. ${ }^{(6)}$

\section{Magnetic resonance imaging}

On T1-weighted images (WI), the normal spleen has a homogeneous low signal intensity that is slightly less than that of the liver and muscle. On T2-WI, signal intensity is higher than that of the liver parenchyma. Splenic signal intensity, however, varies with patient age. (1) In the newborn, white pulp is not yet matured, resulting in a more hypointense signal intensity than the normal liver parenchyma on T2-WI and more isointense signal on T1-WI. Within the first months of life, the imaging characteristics evolve to the normal adult pattern. Pulse sequences used for MR imaging of the spleen are similar to those used for routine liver imaging (Table I). MR images obtained after dynamic administration of a gadolinium contrast agent may show an inhomogeneous pattern of enhancement during the arterial phase, similar to the contrast enhancement pattern on CT (Fig. 3).

${ }^{1}$ Department of Radiology, Leuven University Hospital, Leuven, ${ }^{2}$ Department of Radiology, Antwerp University Hospital, Edegem, ${ }^{3}$ Department of Radiology, AZ Sint-Maarten, Duffel-Mechelen, ${ }^{4}$ Faculty of Medicine and Health Sciences, Ghent University, Gent, Belgium

Correspondence: Dr Thomas Vancauwenberghe, Department of Radiology, Leuven University Hospital, Herestraat 49, 3000 Leuven, Belgium.

thomas.vancauwenberghe@uzleuven.be 

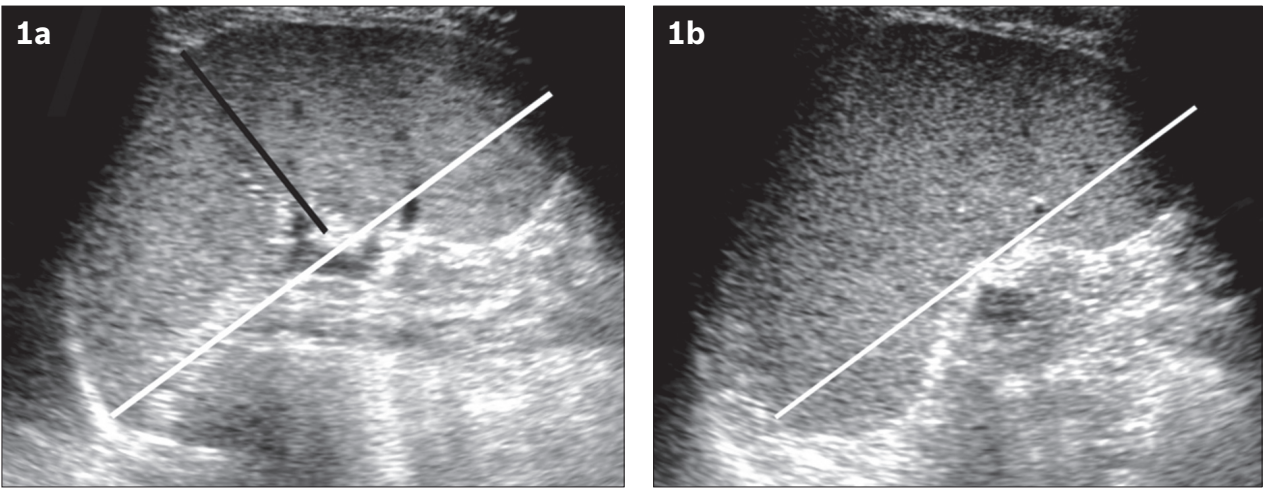

Fig. 1 Normal spleen on US. (a) Coronal and (b) axial views of the left upper quadrant show a normal spleen. The black line represents the splenic width, the white line in (a) represents the splenic length and the white line in (b) indicates the splenic depth.
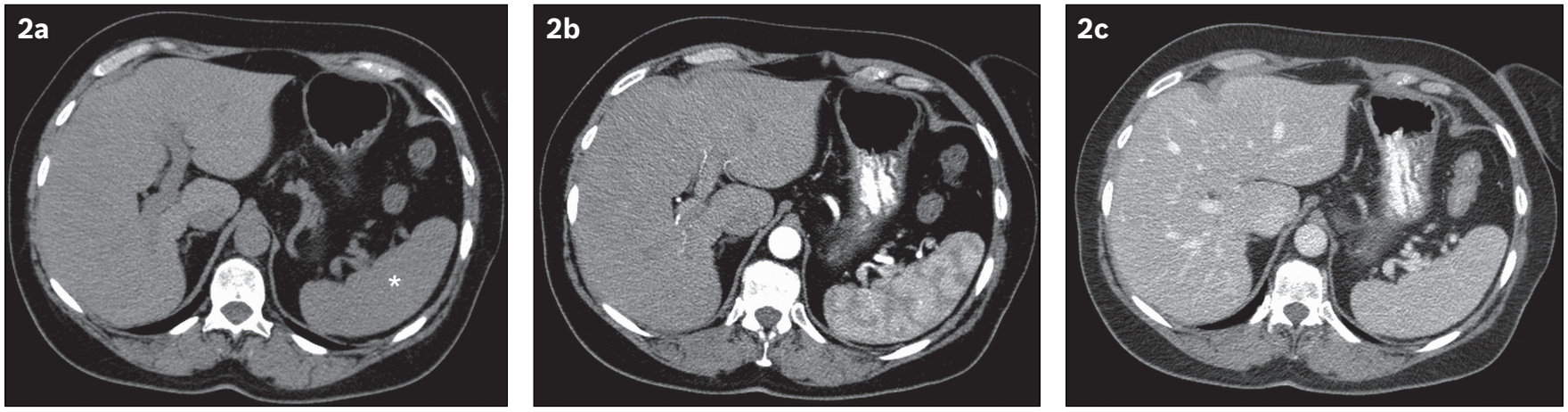

Fig. 2 Normal enhancement pattern of the spleen on CT. Axial CT images (a) before and (b \& c) after intravenous administration of iodinated contrast material show heterogeneous enhancement of the splenic parenchyma during the arterial phase (as seen in Fig. $2 b$ ). (c) In the portal phase, there is homogeneous enhancement.
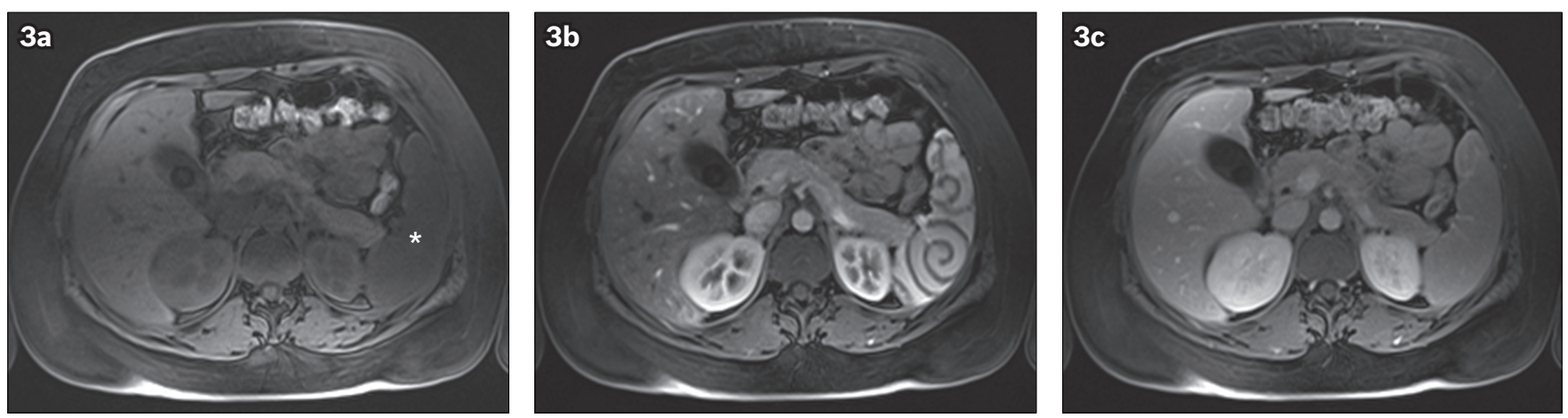

Fig. 3 Normal enhancement pattern of the spleen on MR imaging. (a) Fat-suppressed T1-W image shows the spleen (asterisk) as being isointense to slightly hypointense to the muscle. (b) During the arterial phase after intravenous gadolinium contrast enhancement, the spleen shows a serpentinecordlike pattern. (c) In the portal phase, there is uniform enhancement throughout the spleen.

\section{Table I. Imaging sequences used for evaluation of the spleen.}

- Axial and coronal ultrafast spin echo T2-weighted imaging

- Axial gradient-echo (GRE) T1-weighted chemical shift in-phase and out-of-phase imaging performed during a breath hold

- Axial three-dimensional GRE breath-hold sequence with pre-contrast and dynamic gadolinium-enhanced imaging

- Optional: Axial GRE T2*-weighted imaging for detecting haemosiderin

\section{SIGNIFICANT SPLENIC VARIATIONS}

\section{Splenic clefts, notches and lobules}

Splenic lobules are remnants of fetal splenic lobulation, persisting in adult life as variations in normal shape. They are most commonly seen along the medial part of the spleen and are sometimes supplied by a branch of the splenic artery. ${ }^{(7)}$ Splenic notches or clefts in the splenic borders are common and represent remnants of the grooves that separated the fetal lobules (Fig. 4). They typically have sharp borders, and can be as deep as $2-3 \mathrm{~cm} .{ }^{(6)}$ Splenic lobules have no clinical significance, but they should not be misinterpreted as splenic laceration in patients with a history of abdominal trauma. Absence of free abdominal or perisplenic fluid and normal splenic enhancement are key imaging features that differentiate these variants from an area of laceration.

\section{Accessory spleen}

Accessory spleens are also known as surnumerary spleens, splenunculi or splenules. They represent normal splenic tissue 


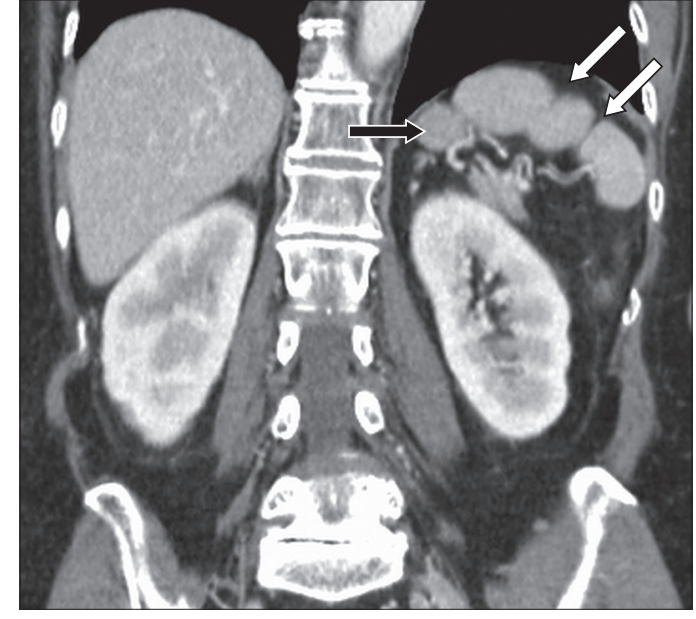

Fig. 4. Splenic lobulation. Coronal contrast-enhanced CT image shows a splenic lobule (black arrow) and two clefts more laterally (white arrows).

separated from the main body of the spleen, originating from nonfusion of the splenic "anlage" and being either solitary or multifocal. ${ }^{(8,9)}$ The size varies from a few millimetres to several centimetres. Accessory spleens are seen in 10\%-15\% of patients, and are usually located near the splenic hilum, the tail of the pancreas, and the gastrosplenic or splenorenal ligaments (Fig. 5). However, they can also be found anywhere in the peritoneal cavity. ${ }^{(1)}$ Because of the close relationship in the development of the spleen and left gonadal anlage, accessory spleens can rarely be demonstrated near the left ovary or within the scrotum, and this is referred to as 'splenogonadal fusion'. ${ }^{(3)}$ On all imaging modalities, an accessory spleen shows similar imaging characteristics as a normal spleen, because it consists of normal splenic parenchyma. ${ }^{(6)}$ Recognising and reporting spenules is important, as they may be responsible for disease recurrence after splenectomy for haematologic or autoimmune disorders. ${ }^{(7)}$ Scintigraphy with Tc-99m sulfur colloid or denatured red cells is the most sensitive technique in detecting splenules. ${ }^{(3)}$

\section{Asplenia and polysplenia}

Complete absence of the spleen ('asplenia') or multiple discrete spleens ('polysplenia') are congenital syndromes that are associated with anomalies in viscero-atrial situs. In the management of these syndromes, it is important to search for associated abdominal and thoracic malformations. It should also be noted that in reality, these syndromes are very complex, since they do not have a fixed set of characteristics that are present in all cases. ${ }^{(10)}$ Polysplenia is usually diagnosed in early childhood because of cardiac anomalies. Most patients die by the age of 5 years. ${ }^{(7)}$ The spleens are usually equally sized and may be left- or right-sided, along the greater curvature of the stomach (Fig. 6). Sometimes there are multiple small spleens adjacent to one or two large spleens. ${ }^{(11)}$ Asplenia is associated with severe congenital heart disease, accounting for a very high mortality rate of up to $95 \%$ in the first year of life. (1) The spleen is absent in virtually all patients with asplenia. Rarely, a rudimentary spleen may be detected. ${ }^{(10)}$

\begin{abstract}
Splenosis
Splenosis represents ectopic splenic peritoneal implants. ${ }^{(12)}$ In contrast to accessory spleens, splenosis is an acquired condition secondary to seeding and implantation of splenic cells after splenic trauma or splenectomy. ${ }^{(7,13)}$ As with accessory and ectopic spleens, such implants consist of functional splenic tissue, which provides some protection against infection. Usually the implants are multiple, measuring from a few millimetres up to several centimetres in diameter. Occasionally, they occur in extraperitoneal locations, such as the chest. US, CT and MR imaging can be used to detect splenosis. On all these imaging modalities, splenosis shows the same imaging characteristics as a normal spleen. Recognising and reporting splenules is important, as they may mimic mass lesions such as metastatic adenopathy or lymphoma. As with accessory spleens, scintigraphy with Tc-99m sulfur colloid or denatured red cells is the most sensitive technique for detecting ectopic splenic peritoneal implants. $^{(3)}$
\end{abstract}

\section{SPLENIC CYSTS}

Histopathologically, cystic lesions of the spleen may be classified as primary (or true cysts) and secondary (or false cysts). While true cysts have an epithelial cellular lining, the cyst wall of false cysts is composed of fibrous tissue. Although reliable differentiation between true and false cysts by imaging is usually not possible and is more of academic interest than clinical importance, there are certain imaging features that may suggest a presumptive diagnosis (Table II). ${ }^{(3)}$ Distinguishing a benign cystic lesion from a malignant lesion is, however, more important than differentiating between a true and false cyst. The absence of wall-thickening, intralesional solid components or contrast-enhancement is in favour of benign lesions. ${ }^{(14)}$

\section{Congenital cysts}

Congenital cysts, also called epidermoid or epithelial cysts, comprise approximately $10 \%$ of all true splenic cysts worldwide. ${ }^{(3)}$ On US, most cysts are unilocular and homogeneously anechoic, with a thin wall (Fig. 7a). On CT, these cysts appear as well-circumscribed, hypodense and nonenhancing lesions (Fig. 7b). On MR imaging, splenic cysts are hypointense on T1-WI and strongly hyperintense on T2-WI. Signal intensity on T1-WI may be increased due to proteinaceous or haemorrhagic content. $^{(15)}$

\section{False cysts}

False cysts account for approximately $80 \%$ of all splenic cysts. ${ }^{(16)}$ They result from either a previous trauma, infection or infarction. ${ }^{(17)}$ On imaging, false cysts tend to be small in size, well defined, and mostly multilocular. They are usually located close to the splenic capsule. False cysts may be inhomogeneous due to intralesional debris. On CT, wall calcifications are seen in 50\% of cases (Fig. 8). ${ }^{(14)} \mathrm{MR}$ imaging typically shows a very high signal on T2-WI and variable signal intensity on T1-WI, depending on the lesion content (either serous, haemorrhagic, proteinaceous or necrotic). ${ }^{(15,16)}$ 



Fig. 5 Accessory spleen adjacent to the pancreatic tail, mimicking a pancreatic mass. (a) Axial contrast-enhanced CT; (b) axial T1-W; and (c) T2-W images show a focal mass (arrows) adjacent to the pancreatic tail, with similar signal intensity and enhancement pattern as the spleen, consistent with an accessory spleen.

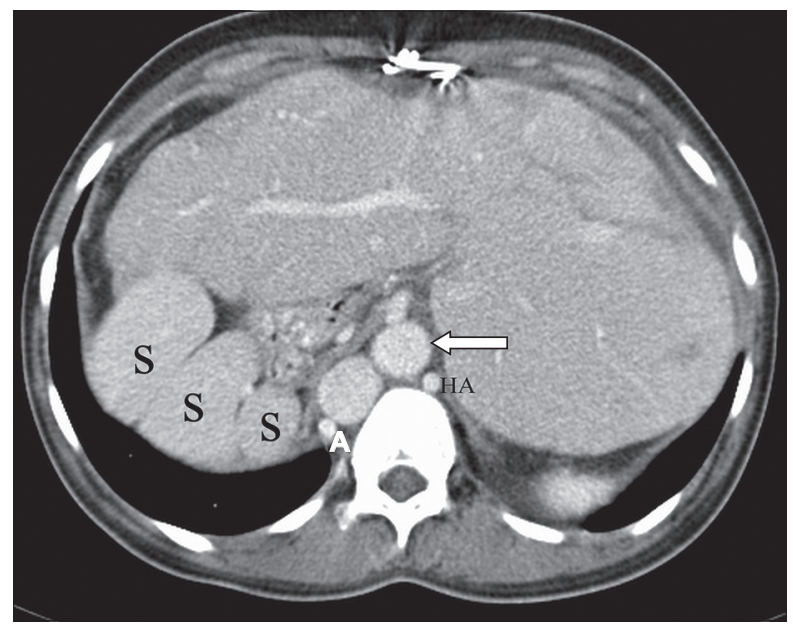

Fig. 6 Polysplenia in a 37-year-old woman. Axial contrast-enhanced CT image shows abdominal situs inversus with multiple round splenules (S) in the right upper quadrant, lateral to the stomach. The liver is left-sided and enlarged. The intrahepatic segment of the inferior vena cava (arrow) is on the left of the aorta. Note the slightly enlarged azygous vein (A) and hemiazygous vein $(\mathrm{HA})$.

Table II. Useful differential diagnostic criteria for imaging characterisation of splenic cysts.

\begin{tabular}{llll}
\hline Parameter & $\begin{array}{l}\text { Epidermoid } \\
\text { cysts }\end{array}$ & $\begin{array}{l}\text { Parasitic } \\
\text { cysts }\end{array}$ & $\begin{array}{l}\text { False } \\
\text { cysts }\end{array}$ \\
\hline $\begin{array}{l}\text { Wall calcifications } \\
\text { Cystic lesion } \\
\text { Single }\end{array}$ & Rare & Sometimes & Frequent \\
$\begin{array}{l}\text { Multiple } \\
\text { Loculation }\end{array}$ & Frequent & Sometimes & Frequent \\
Unilocular & Frequent & Rare & Frequent \\
Multilocular & Rare & Frequent & Rare \\
\hline
\end{tabular}

\section{BENIGN TUMOURS}

\section{Haemangioma}

Haemangiomas are vascular proliferations composed of channels filled with slow-flowing blood. ${ }^{(1)}$ Depending on the size of these channels, haemangiomas are divided into capillary and cavernous types. ${ }^{(3)}$ On US, a capillary haemangioma appears as a hyperechoic mass, whereas a cavernous haemangioma is heterogeneously hypoechoic, sometimes with calcifications or multiple cystic areas. ${ }^{(18)}$ Abundant blood flow may be demonstrated with colour Doppler. On unenhanced CT, most haemangiomas are iso- to hypodense relative to normal splenic tissue. After contrast administration, cavernous haemangiomas show diffuse mottled or peripheral nodular enhancement extending toward the centre. ${ }^{(19)}$ Capillary haemangiomas show homogeneous contrast enhancement. ${ }^{(1)}$ Calcifications, either peripheral curvilinear or mottled centrally, may occur (Fig. 9a). ${ }^{(18)}$

On MR imaging, haemangiomas are either hypo- or isointense on T1-WI and heterogeneously hyperintense on T2-WI. ${ }^{(3)}$ A high signal intensity on T1-WI suggests the presence of subacute haemorrhage or proteinaceous content. ${ }^{(16)}$ Superimposed infarction and thrombosis in larger haemangiomas may cause a variable MR imaging appearance. ${ }^{(20)}$ In the latter case, differentiation from malignant disease may be difficult. As on CT, cavernous haemangiomas show peripheral enhancement extending toward the centre (Figs. 9b-d). Capillary haemangiomas, however, have more homogeneous contrast enhancement.

\section{Lymphangioma}

Lymphangiomas are malformations of the lymphatic system that consist of various sized cystic dilatations, containing lymph. Multiple lymphangiomas may be part of systemic lymphangiomatosis. Three histologic subtypes have been identified, depending on the size of the lymph channels, namely capillary, cavernous and cystic lymphangiomas. ${ }^{(18)}$

On US, typical lymphangiomas are multilocular, thinwalled, hypoechoic cyst-like lesions of different sizes, located close to the splenic capsule. Hyperechoic septa and intralocular echogenic debris may be detected. ${ }^{(18)}$ On CT, tiny curvilinear peripheral calcifications may be occasionally identified. There is no contrast enhancement. ${ }^{(19)}$ On MR imaging, multilocular lesions are homogeneously hyperintense on $\mathrm{T} 2-\mathrm{WI}$ and hypointense on T1-WI (Fig. 10). Areas of high signal intensity may be seen on $\mathrm{T} 1-\mathrm{Wl}$, owing to intracystic proteinaceous content or internal haemorrhage. ${ }^{(3)}$ Fibrous septa appear as hypointense bands on both T1- and T2-WI. MR imaging is more sensitive than CT in detecting solid elements in the cystic lumen. ${ }^{(20)}$ The latter finding is rare but important, since malignant degeneration should be excluded in these cases. 



Fig. 7 Congenital cyst. (a) US image of a 20-year-old woman with chronic abdominal pain shows a thin-walled anechoic lesion near the splenic hilum (arrow). (b) Axial contrast-enhanced CT image in the same patient shows a round, thin-walled hypodense lesion (arrow).

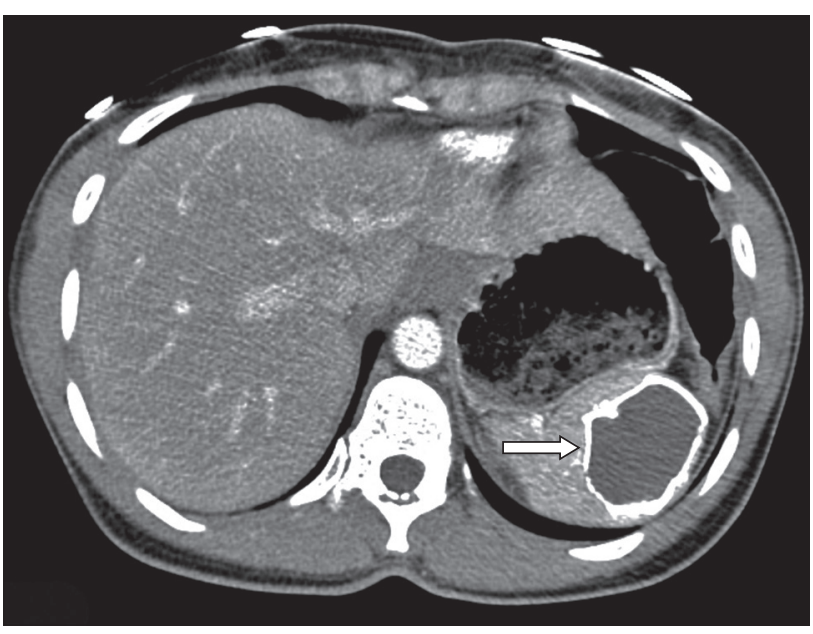

Fig. 8 False cyst. Contrast-enhanced CT image shows a cystic unilocular, hypodense splenic mass with wall calcifications (arrow).

\section{Littoral cell angioma}

Littoral cell angioma represents a very rare benign vascular splenic tumour composed of anastomosing vascular channels. ${ }^{(18)}$ Most patients are asymptomatic. The US appearance varies from splenomegaly with a mottled echotexture to multiple solid iso-, hypo- or hyperechoic nodules (Fig. 11a). ${ }^{(2)}$ When the lesions are hyperechoic, differentiation from haemangiomas may be difficult. ${ }^{20)}$ On unenhanced $\mathrm{CT}$, they present as multiple hypoattenuating nodules of different sizes. Sometimes a solitary large lesion is seen (Fig. 11b). They show prolonged contrast enhancement due to their histological structure of multiple vascular channels on $\mathrm{CT}$ as MR imaging. ${ }^{(14)} \mathrm{A}$ littoral cell angioma is of marked low signal intensity on both T1- and T2-WI, due to the haemosiderin content. However, as haemosiderin is not always present, the absence of low signal foci does not exclude the diagnosis of littoral cell angioma. ${ }^{(21)}$

\section{MALIGNANT TUMOURS \\ Lymphoma}

Both Hodgkin and non-Hodgkin lymphomas represent the most common malignant neoplasms of the spleen. ${ }^{(14)}$ Primary and secondary splenic involvement may occur. Primary involvement is rare, representing less than $1 \%$ of all lymphomas, most of which are non-Hodgkin lymphomas. ${ }^{(18)}$ In secondary involvement, extrasplenic disease such as retroperitoneal lymph node enlargement often suggests the correct diagnosis. ${ }^{(19)}$

US of splenic lymphoma has a variable imaging appearance, ranging from a normal appearance, homogeneous or heterogeneous splenomegaly, multiple small (miliary) hypoechoic nodules (Fig. 12a) to a solitary mass or multiple large hypoechoic masses $(1-10 \mathrm{~cm}) .{ }^{(19)}$ The nodules show internal vascularity and may extend beyond the splenic capsule. ${ }^{(21)}$ Less commonly, a target appearance and intralesional calcifications are seen. ${ }^{(22)}$ Hypoechoic splenic deposits may become iso- to hyperechoic as they regress with treatment, due to fibrosis (Fig. 12b). ${ }^{(22)}$ On CT, nodules are of low attenuation, and rarely enhance; therefore, the best lesion conspicuity is demonstrated after contrast administration. ${ }^{(18)}$ Necrosis, occurring in large lesions, may cause an irregular cystic appearance, resembling an abscess (Fig. 13). ${ }^{(19)}$ Variation in the size of lesions is more indicative of lymphomatous involvement rather than multifocal abscesses. Infarction of the spleen as a result of lymphoma is not uncommon. Lymphomas are typically hypointense or nearly isointense to normal splenic parenchyma on T1-WI and hyperintense on T2-WI.

\section{Metastasis}

Splenic metastases are seen in $2 \%-9 \%$ of patients with metastatic end-stage cancer. ${ }^{(14)}$ Haematogeneous spread from the breast, lung, ovary, stomach, prostate carcinoma and cutaneous melanoma are most common. ${ }^{(15)}$ Evidence of metastatic spread, such as metastasis to the liver or other organs, is usually present. Peritoneal implants on the surface of the spleen are frequently seen in patients with primary tumours of the ovary, adenocarcinoma of the gastrointestinal tract and pancreatic cancer (Fig. 14).(15)

On US, metastases appear hypoechoic and occasionally mixed or hyperechoic. Cystic change is seen when necrosis occurs or due to the mucinous nature of the primary tumour (e.g. ovarian carcinoma). ${ }^{(19)}$ On CT, splenic metastases appear as well-delineated, low-attenuation cystic or solid masses. ${ }^{(15)}$ Calcification is unusual, unless the primary tumour is a mucinous adenocarcinoma. Most lesions show peripheral or septal enhancement (Fig. 15). ${ }^{(15,16)}$ Peritoneal metastases cause scalloping of the splenic surface. ${ }^{(15)} \mathrm{MR}$ imaging shows moderate hyperintensity on $\mathrm{T} 2-\mathrm{WI}$ and isointensity on $\mathrm{T} 1-\mathrm{WI}$, 



Fig. 9 Cavernous haemangioma. (a) Axial unenhanced CT image shows an amorphous calcification anterior to the spleen (arrow). Axial T1-W images after intravenous administration of gadolinium contrast in the (b) arterial; (c) portovenous; and (d) delayed phases show the calcification to be centrally located in a rounded lesion, representing a cavernous haemangioma. Note the typical irregular peripheral enhancement in the arterial phase, extending in a centripetal manner during the portovenous phase, and pooling in the delayed phase.
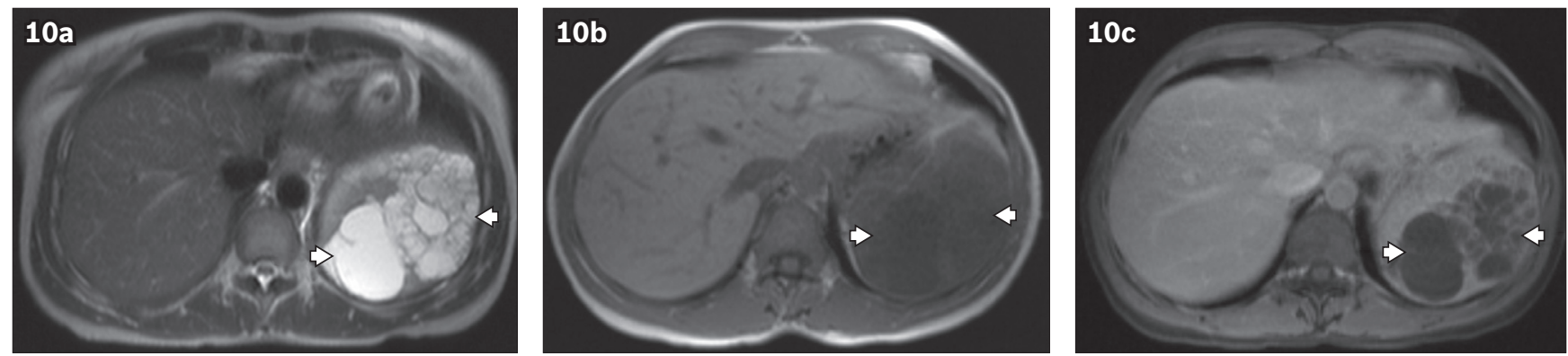

Fig. 10 Lymphangiomatosis. (a) Axial T2-W MR image shows multiple, multilocular cyst-like lesions (arrowheads). (b) T1-W MR image shows the cystic lesions appearing hypointense (arrowheads). (c) T1-W MR image after administration of gadolinium contrast, in the delayed phase, shows enhancement of the septa.

relative to splenic parenchyma. The presence of blood products or other paramagnetic substances, such as melanin in metastatic melanoma, may result in high signal intensity on T1-WI. ${ }^{(16)}$

\section{Angiosarcoma}

Splenic angiosarcoma is the most common malignant primary vascular neoplasm of the spleen. ${ }^{(20)}$ Symptoms include weight loss, abdominal pain, malaise, fever, a palpable abdominal mass and hypovolemic shock due to splenic rupture. ${ }^{(21)}$ On imaging, splenic angiosarcoma manifests as a poorly circumscribed heterogeneous mass, with internal areas of haemorrhage and necrosis. ${ }^{(21)}$ Heterogeneous signal before and after contrast administration reflects the haemorrhagic nature of the tumour. ${ }^{(14)}$ Differentiation from a large haemangioma with superimposed infarction and thrombosis may be difficult. ${ }^{(20)}$

\section{SPLENIC INFARCTION}

Splenic infarcts are common and can be of either arterial or venous origin. Arterial infarction occurs secondary to occlusion of the splenic artery ('global infarction') or one of its noncommunicating 

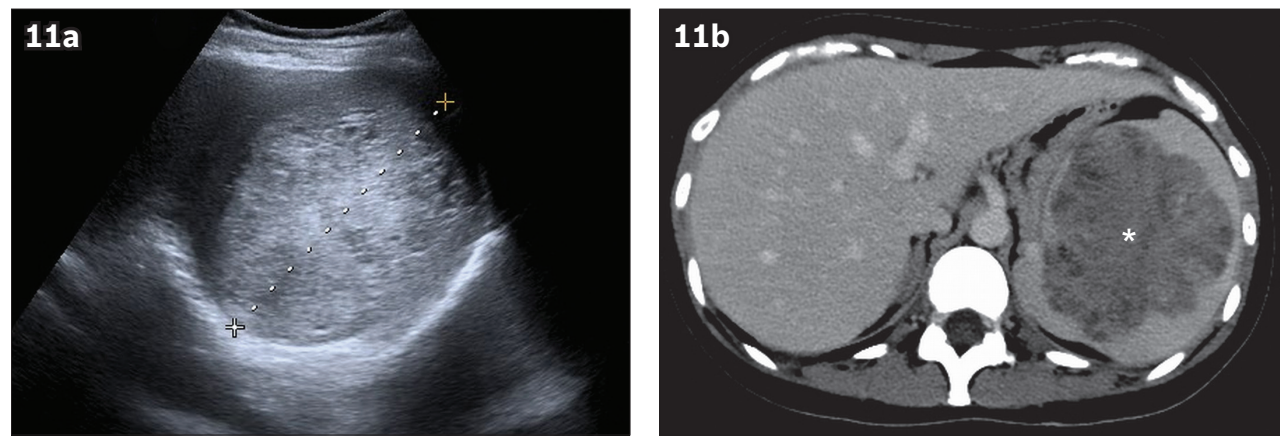

Fig. 11 Littoral cell angioma in a 35-year-old woman with anaemia and thrombocytopenia. (a) Axial US image of the spleen shows splenomegaly with a focal heterogeneous hyperechoic mass. (b) Axial contrast-enhanced CT image in the portovenous phase confirms an enlarged spleen, containing an irregularly delineated heterogeneous enhancing lesion (asterisk).


Fig. 12 Multifocal splenic involvement in lymphoma. (a) US image shows several small hypoechoic splenic deposits in a patient with histologically proven mantle cell lymphoma (arrows). (b) Axial contrast-enhanced CT image acquired during the portovenous phase shows multiple low-attenuation lesions within an enlarged spleen (arrowheads). Variation in the size of lesions is more indicative of lymphomatous involvement rather than multifocal abscesses.
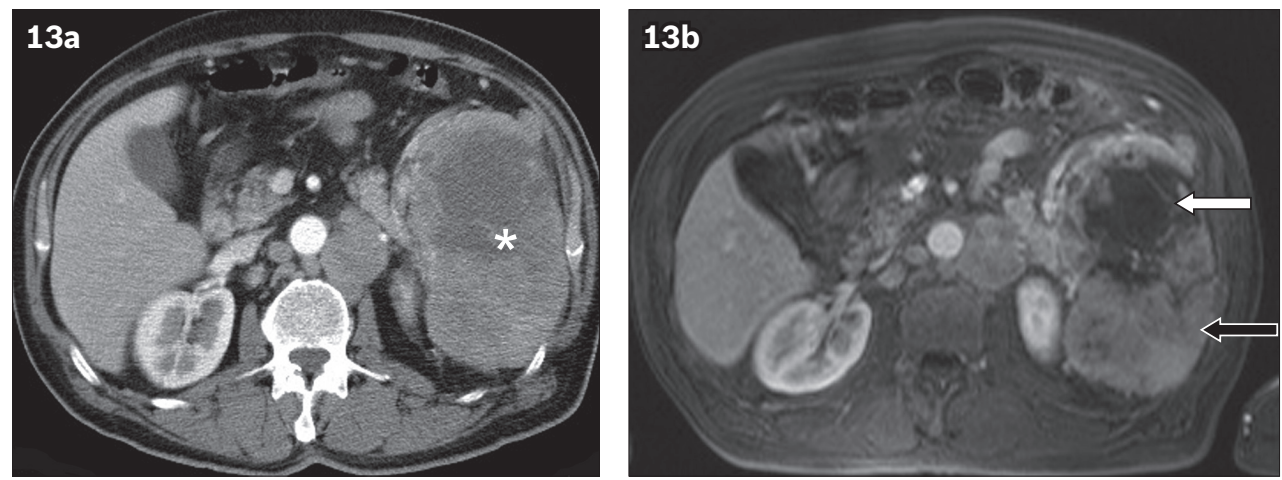

Fig. 13 Splenic involvement in lymphoma. (a) Axial contrast-enhanced CT image acquired during the arterial phase shows a large heterogeneous lesion (asterisk). (b) Axial T1-W image obtained after the administration of gadolinium contrast in the arterial phase shows viable (black arrow) and necrotic (white arrow) parts of the tumour. Note also that the normal parenchyma is almost entirely replaced by the lesion.

branches ('segmental infarction'). ${ }^{(2)}$ Thrombosis of the splenic sinusoids may cause venous infarction. ${ }^{(16)}$ Complications of infarcts include splenic rupture, splenic pseudocyst formation, haemorrhage and superimposed infection with abscess formation. ${ }^{(3)}$

On US, acute splenic infarcts typically appear as wedgeshaped hypoechoic lesions pointing toward the splenic hilum. ${ }^{(1)}$ On non-enhanced CT, infarcts are poorly visualised. After intravenous iodine contrast administration, the typical imaging findings are peripheral, wedge-shaped non-enhancing defects. However, this typical appearance is only present in less than half of all acute splenic infarcts. ${ }^{(2)}$ In less typical cases, infarcts may mimic other splenic lesions, including abscesses or tumours, requiring clinical correlation, or if necessary, percutaneous fine-needle aspiration biopsy. ${ }^{(13)}$ When the entire spleen is infarcted, it results in diffuse splenic hypodensity, leaving a residual rim of enhancing capsule supplied by small capsular vessels. This is known as the 'rim sign'. ${ }^{(13)}$ Chronic infarcts decrease in size, resulting in fibrotic retraction of the splenic capsule (Fig. 16). ${ }^{(2)}$ In the case of haemoglobinopathy, 


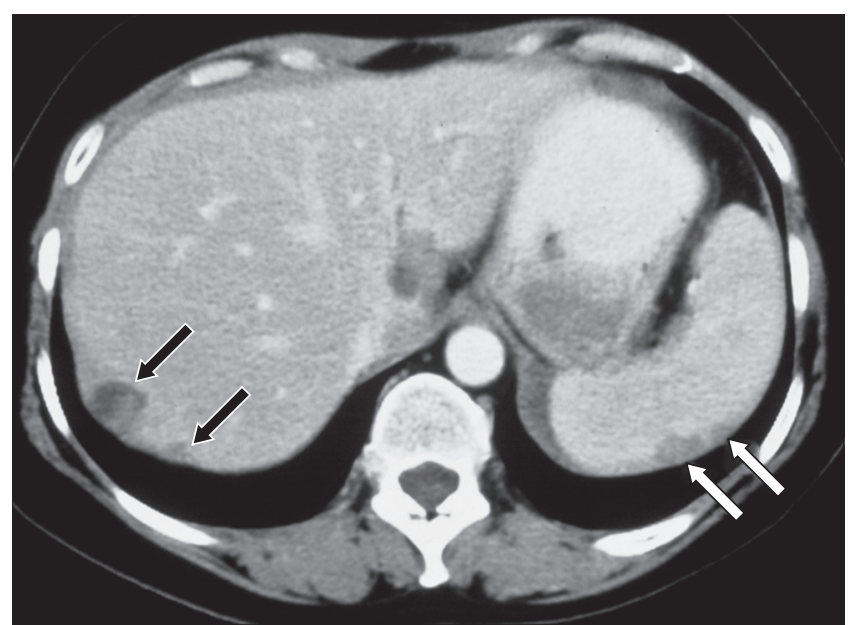

Fig. 14 Peritoneal splenic implant metastases in a patient with metastatic ovarian carcinoma. Axial contrast-enhanced CT image in the portovenous phase shows two well-circumscribed polylobulated and hypodense lesions on the dorsal surface of the spleen (white arrows). Note two more similar lesions on the dorsal surface of the liver (black arrows)

curvilinear or punctate calcifications may occur as sequelae of repeated infarctions (Fig. 17). On MR imaging, the signal intensity of infarcted areas varies, depending on the age of the lesion and the degree of haemorrhage. ${ }^{(2)}$ Recent haemorrhagic areas are of high signal intensity on T1-WI. In the subacute or chronic stage liquefaction may occur, resulting in a decrease of signal on T1-WI and an increase on T2-WI. The contrast enhancement pattern on MR imaging is similar to that on CT.

\section{INFECTIOUS DISEASES}

Splenic abscess is a rather uncommon lesion. ${ }^{(23)}$ However, its incidence is increasing due to the widespread use of immunosuppressive drugs and chemotherapy. Splenic abscesses may be pyogenic, parasitic, fungal or tuberculous. ${ }^{(23)}$

\section{Pyogenic abscesses}

On US, abscesses appear as ill-defined hypo- or anechoic lesions, depending on the degree of proteinaceous fluid and necrosis. Debris, fluid levels and internal septations of varying thickness may be seen. ${ }^{(3)}$ Intralesional gas, causing echogenic foci with 'dirty' shadowing, is highly suggestive of pyogenic infection, although the majority of splenic abscesses do not contain air. ${ }^{(3)}$ On $\mathrm{CT}$, pyogenic abscesses are irregularly marginated lesions, with inhomogeneous low attenuation (Fig. 18). ${ }^{(3)} \mathrm{CT}$ is the most sensitive method of identifying small amounts of intralesional gas. ${ }^{(16)} \mathrm{On}$ MR imaging, splenic pyogenic abscesses appear as lesions of fluid signal intensity, being hypointense to normal splenic tissue on T1-WI and hyperintense on T2-WI. Peripheral enhancement can be seen on both contrast-enhanced CT and MR imaging. ${ }^{(3,24)}$

\section{Fungal abscesses}

Fungal abscesses occur most commonly in immunocompromised individuals. ${ }^{(15)}$ The most common infecting organisms are Candida albicans, Aspergillus fumigatus and Cryptococcus neoformans. ${ }^{(3)}$ Fungal abscesses are typically multifocal and only a few millimetres in diameter. ${ }^{(19)}$ On US, they are seen as lesions with a 'target' or 'bull's-eye' appearance due to a central hyperechoic inflammatory core surrounded by hypoechoic fibrotic tissue. ${ }^{(3)}$ The hyperechoic portion may become partially necrotic and hypoechoic, resulting in a 'wheel-in-a-wheel' pattern. ${ }^{(3)}$ CT typically demonstrates multiple low-attenuation lesions, usually 5-10 mm in size (Fig. 19). Occasionally, a central hyperdense focus ('target appearance') may be demonstrated on CT. On MR imaging, splenic fungal lesions demonstrate intermediate signal intensity on $\mathrm{T} 1-\mathrm{WI}$ and high signal intensity on T2-WI, relative to normal splenic tissue. ${ }^{(15)}$ After intravenous contrast administration, there is either no enhancement or only subtle ring-like enhancement. ${ }^{(15)}$ Due to this frequent lack of contrast enhancement and their small size, fungal abscesses may easily be overlooked on cross-sectional imaging.

\section{Tuberculous infection}

There are two major morphological subtypes of hepatosplenic tuberculosis - micronodular and macronodular. ${ }^{(25)}$ The micronodular (or miliary form) is the most common. On US, the micronodular form of hepatosplenic tuberculosis usually presents as a hyperechoic splenomegaly ('bright spleen'). Rarely, multiple tiny hypoechoic and occasionally, hyperechoic focal lesions, are seen (Fig. 20a). ${ }^{(25)}$ The macronodular form may be either solitary or multiple, with a diameter of $1-3 \mathrm{~cm}$. On US, hypoechogenicity is the rule, although some lesions may be hyperechoic. These differences in echogenicity probably represent a different phase of disease - the hyperechoic lesions represent an earlier stage, whereas the echo-poor lesions correspond to the stage of caseation necrosis. ${ }^{(25)}$

Although micronodular lesions are usually below the resolution of $\mathrm{CT}$, tiny low-density foci are occasionally seen throughout the spleen (Fig. 20b). Calcifications may occur in late stage disease. ${ }^{(25)}$ However, splenic calcifications are nonspecific and may arise in many other diseases. Abdominal lymphadenopathy with low central attenuation due to caseous necrosis, hepatomegaly, high-attenuation ascites (25-45 HU) with nodular peritoneal thickening, pleural effusions and focal hepatic lesions are the common associated findings. ${ }^{(15)} \mathrm{MR}$ imaging findings of focal micro- and macronodular tuberculous lesions are usually nonspecific, being hypointense on T1-WI and hyperintense on T2-WI. ${ }^{(25)}$ Both CT and MR imaging may show subtle peripheral enhancement.

\section{INFLAMMATORY DISEASE}

\section{Sarcoidosis}

Sarcoidosis is a multisystem granulomatous disease of unknown aetiology that is histologically characterised by multiple nonspecific non-caseating granulomas. ${ }^{(3)}$ The lung, mediastinal and hilar lymph nodes are most frequently affected. The spleen is involved in up to $59 \%$ of cases. ${ }^{(3)}$ On US, splenic sarcoidosis most commonly presents as a homogeneous enlarged spleen, with associated lymphadenopathy in the splenic hilum and retroperitoneum. A nodular form of involvement is seen in only $15 \%$ of cases. ${ }^{(21)}$ These nodules may be detected as discrete hypoechoic lesions of variable sizes, ranging from $1 \mathrm{~mm}$ to $3 \mathrm{~cm}$ 

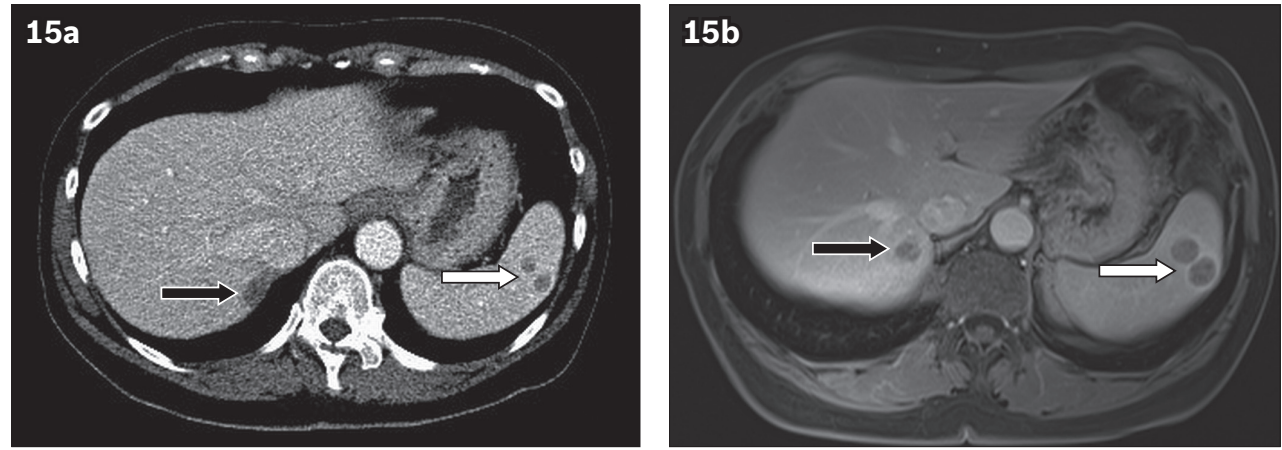

Fig. 15 Splenic metastatic disease from lung carcinoma. Axial contrast-enhanced (a) CT and (b) T1-W images show two low-attenuation lesions with subtle peripheral enhancement (white arrow). Note a similar lesion posteriorly in the liver (black arrow), representing a liver metastasis.



Fig. 16 Splenic infarction in a 79-year-old man with known atrial fibrillation. Coronal contrast-enhanced CT image shows a well-demarcated, wedgeshaped region of decreased enhancement with parenchymal loss and retraction of the splenic capsule, indicating the chronic nature of the infarction (arrow).

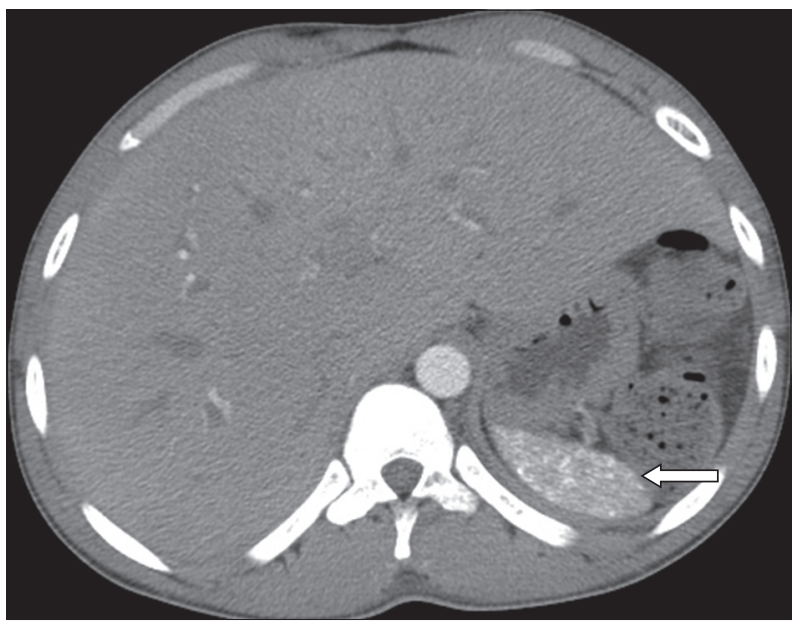

Fig. 17 Autosplenectomy in an adult with sickle cell disease. Axia contrast-enhanced CT image shows a small, shrunken spleen, with diffuse calcifications due to repeated micro-infarctions (arrow)

(Fig. 21a). ${ }^{(3,21)}$ On CT, these nodules are ill defined and slightly hypodense relative to normal splenic tissue. Hypovascularity is the rule, with only minimal and/or delayed enhancement (Fig. 21b). ${ }^{(21)}$ Hepatic involvement is present in approximately $50 \%$ of patients with splenic sarcoidosis. ${ }^{(19)}$ On MR imaging, nodular sarcoidosis typically has low signal on all MR sequences, and this is most conspicuous on fat-suppressed T2-WI. The nodules are hypovascular on contrast-enhanced images.

\section{SPLENOMEGALY}

Splenomegaly is a common nonspecific manifestation of many disorders. ${ }^{(3)}$ The most common cause is portal hypertension, often

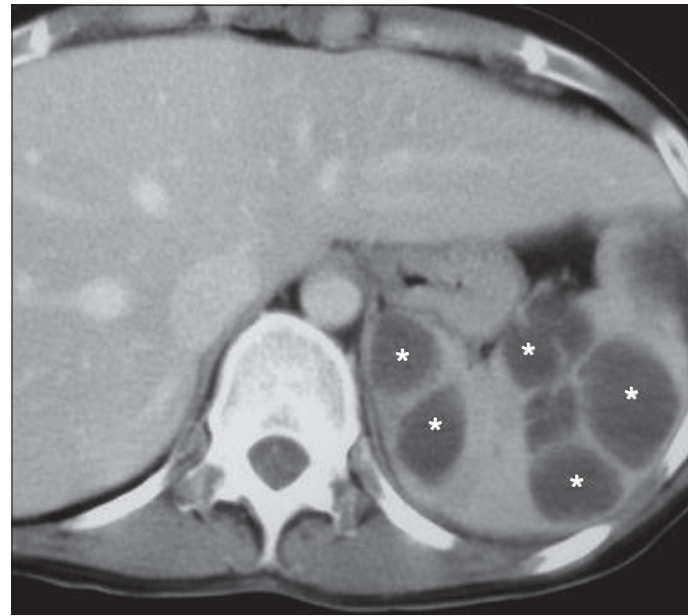

Fig. 18 Pyogenic abscesses. Axial contrast-enhanced CT image acquired during the portal-venous phase shows multiple irregularly marginated nonenhancing lesions (asterisks).

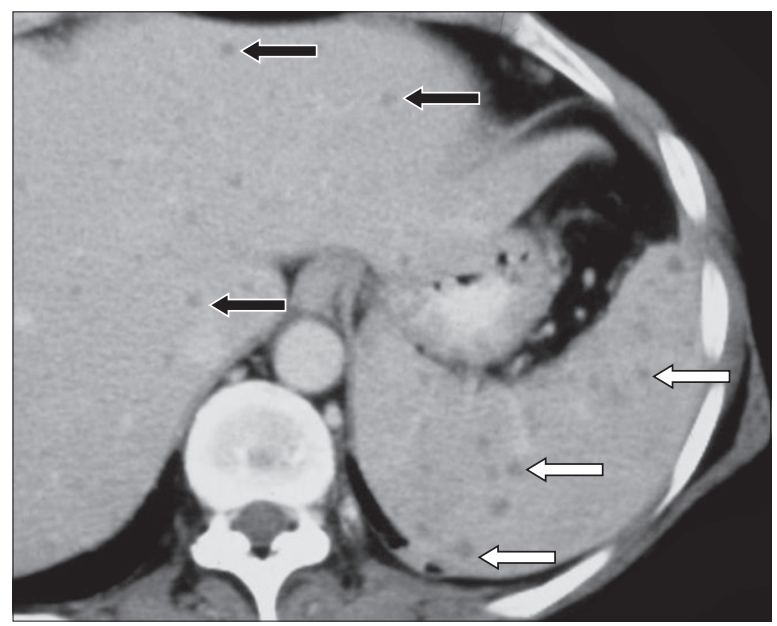

Fig. 19 Fungal abscesses. Axial contrast-enhanced CT image acquired during the portal-venous phase shows multiple small non-enhancing splenic foci (white arrows). Note also multiple similar small non-enhancing foci in the liver, representing fungal liver abscesses (black arrows).

associated with liver cirrhosis. ${ }^{(13)}$ Different imaging modalities can be used to confirm splenomegaly and to search for extrasplenic manifestations of disease, providing clues to the correct cause. ${ }^{(1)}$ Imaging may detect complications, including splenic rupture and infarction (Fig. 22). The upper limit is defined as $15 \mathrm{~cm}$ length (craniocaudal), $10 \mathrm{~cm}$ width (laterolateral) and $6 \mathrm{~cm}$ depth (anteroposterior). ${ }^{(26)}$ The 'splenic index', which is the product of 

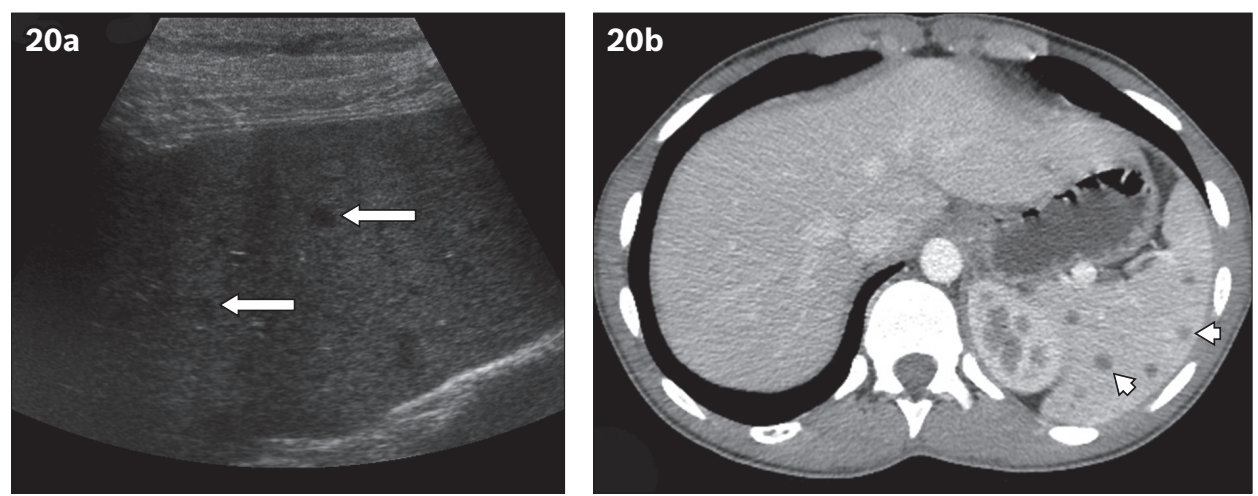

Fig. 20 Splenic tuberculosis. (a) Sagittal US image shows an enlarged spleen with multiple hypoechoic nodules of different sizes (arrows). (b) Contrast-enhanced CT shows widespread hypo-enhancing nodules, representing miliary splenic tuberculosis (arrowheads).
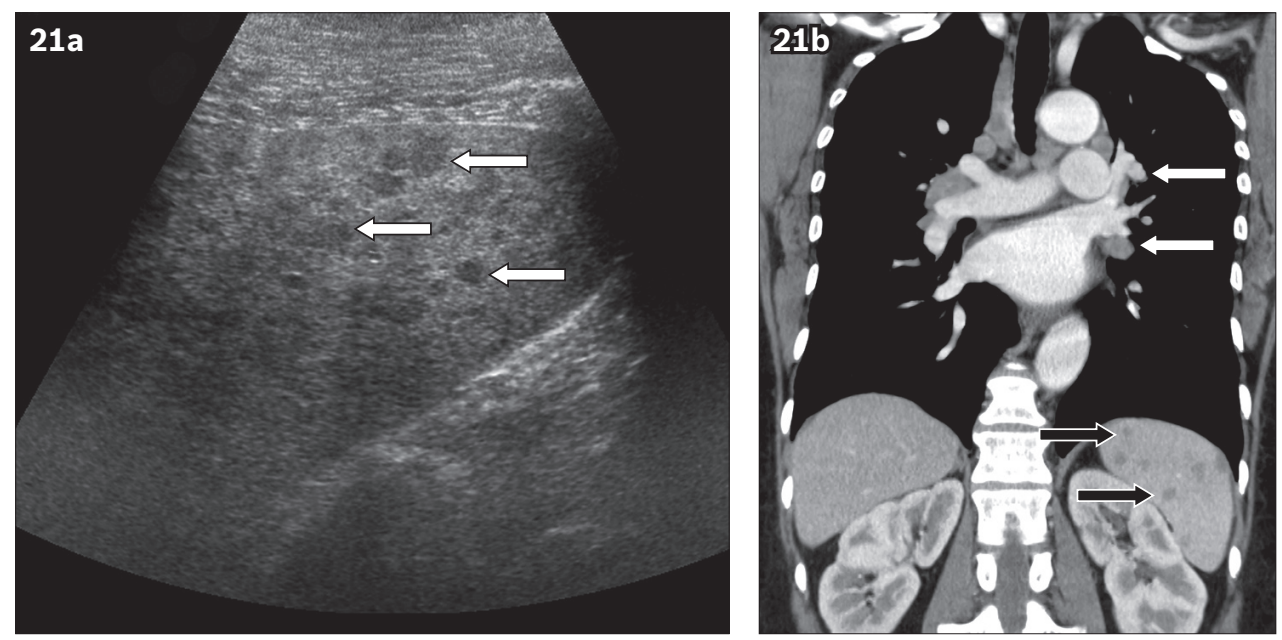

Fig. 21. Splenic sarcoidosis. (a) US image shows a normal-sized spleen with inhomogeneous echotexture and innumerable small hypoechoic lesions (arrows). (b) Coronal contrast-enhanced CT image acquired during the portovenous phase in a 50-year-old man shows multiple well-defined nodules of decreased enhancement throughout the spleen, representing small non-caseating granulomas (black arrows). Note the mediastinal and hilar adenopathies, which provide clues to the diagnosis (white arrows).

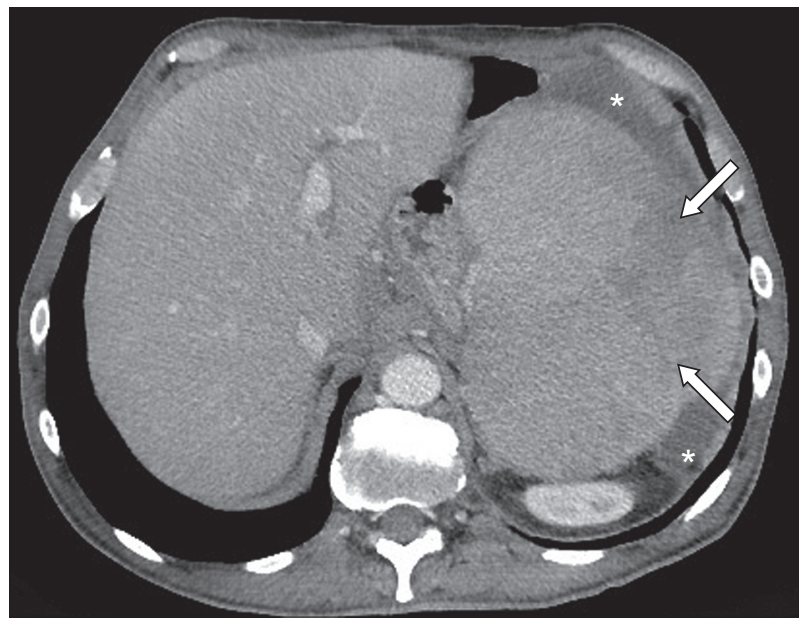

Fig. 22 Splenomegaly complicated with spontaneous rupture. Axial contrast-enhanced CT image shows free perisplenic fluid (asterisks) and an enlarged spleen with a heterogeneous area (arrows), which represents the area of laceration.

the width, depth and length of the spleen, has also been proposed as an indicator for evaluating splenic size. ${ }^{(27)}$ The normal range is $160-440 \mathrm{~cm}^{3}$. ${ }^{(26)}$ Useful rules of thumb for quick assessment of splenic enlargement include extension of the spleen beyond

\section{Table III. Aetiology of Gamna-Gandy bodies.}

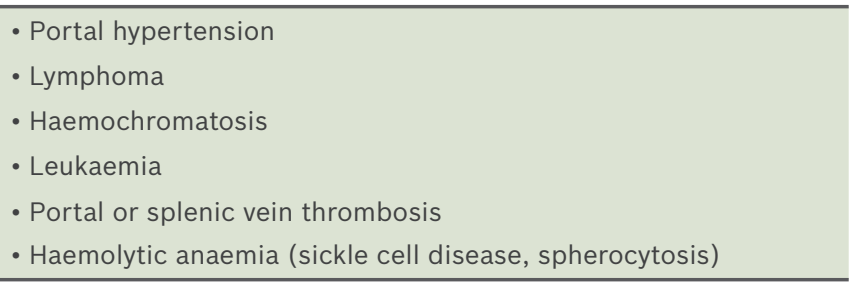

the lower pole of the left kidney, medial extension to the aorta, and loss of its inferomedial concavity. ${ }^{(1,3)}$

\section{Gamna-Gandy bodies}

Gamna-Gandy bodies are small haemorrhages in the spleen caused by different disorders, as summarised in Table III. They are most commonly secondary to portal hypertension. These lesions contain haemosiderin, a variable amount of fibrous tissue and calcium. ${ }^{(2)}$ If the lesions contain sufficient calcium, US may demonstrate hyperechoic areas, with acoustic shadowing. On $\mathrm{CT}$, non-calcified foci appear as multiple, small low-attenuation foci, ${ }^{(2)}$ while calcified lesions appear hyperdense. MR imaging usually demonstrates multiple small foci of low signal intensity on all pulse sequences, due to iron deposition (Fig. 23a).(14) On 



Fig. 23 Gamna-Gandy bodies in a 68-year-old man with liver cirrhosis and portal hypertension. (a) T1-W MR image obtained after administration of gadolinium contrast shows multiple hypointense foci throughout the spleen, representing haemosiderin (arrows). (b) Gradient-echo T1-W image shows the 'blooming artefact' due to the paramagnetic effect of haemosiderin. Note the nodular aspect of the liver parenchyma, which is compatible with cirrhosis.

gradient-echo sequences, 'blooming artefact' is seen due to the paramagnetic effect of haemosiderin (Fig. 23b).

\section{SUMMARY}

The spleen can be affected by a variety of conditions. US, CT and MR imaging are complementary tools for noninvasive characterisation and evaluation of splenic diseases. Many of these conditions can involve the spleen and have similar imaging manifestations. Therefore, imaging findings outside the spleen, along with the patient's clinical history, may help to narrow the differential diagnosis. If uncertainty of a diagnosis persists, percutaneous biopsy may be considered.

\section{REFERENCES}

1. Hilmes MA, Strouse PJ. The pediatric spleen. Semin Ultrasound CT MR 2007; 28:3-11.

2. Vanhoenacker FM, Op de Beeck B, De Schepper AM, et al. Vascular disease of the spleen. Semin Ultrasound CT MR 2007; 28:35-51.

3. Paterson A, Frush DP, Donnelly LF, et al. A pattern-oriented approach to splenic imaging in infants and children. Radiographics 1999; 19:1465-85.

4. Urban BA, Fishman EK. Helical CT of the spleen. AJR Am J Roentgenol 1998; 170:997-1003.

5. Catalano O, Sandomenico F, Vallone P, D'Errico AG, Siani A. Contrastenhanced sonography of the spleen. Semin Ultrasound CT MR 2006; 27:426-33.

6. Fenchel S, Boll DT, Fleiter TR, Brambs HJ, Merkle EM. Multislice helical CT of the pancreas and spleen. Eur J Radiol 2003; 45 Suppl 1:S59-72.

7. Gayer G, Hertz M, Strauss S, Zissin R. Congenital anomalies of the spleen. Semin Ultrasound CT MR 2006; 27:358-69.

8. Dodds WJ, Taylor AJ, Erickson SJ, Stewart ET, Lawson TL. Radiologic imaging of splenic anomalies. AJR Am J Roentgenol 1990; 155:805-10.

9. Mortelé KJ, Mortelé B, Silverman SG. CT features of the accessory spleen. AJR Am J Roentgenol 2004; 183:1653-7.

10. Fulcher AS, Turner MA. Abdominal manifestations of situs anomalies in adults. Radiographics 2002; 22:1439-56.
11. Gayer G, Zissin R, Apter S, et al. CT findings in congenital anomalies of the spleen. Br J Radiol 2001; 74:767-72.

12. Fishman EK, Soyer P, Bliss DF, Bluemke DA, Devine N. Splenic involvement in pancreatitis: spectrum of CT findings. AJR Am J Roentgenol $1995 ; 164: 631-5$

13. Taylor AJ, Dodds WJ, Erickson SJ, Stewart ET. CT of acquired abnormalities of the spleen. AJR Am J Roentgenol 1991; 157:1213-9.

14. Karlo CA, Stolzmann P, Do RK, Alkadhi H. Computed tomography of the spleen: how to interpret the hypodense lesion. Insights Imaging 2013; 4:65-76.

15. Rabushka LS, Kawashima A, Fishman EK. Imaging of the spleen: CT with supplemental MR examination. Radiographics 1994; 14:307-32.

16. Urrutia M, Mergo PJ, Ros LH, Torres GM, Ros PR. Cystic masses of the spleen: radiologic-pathologic correlation. Radiographics 1996; 16:107-29.

17. Ito K, Mitchell DG, Honjo K, et al. MR imaging of acquired abnormalities of the spleen. AJR Am J Roentgenol 1997; 168:697-702.

18. Giovagnoni A, Giorgi C, Goteri G. Tumours of the spleen. Cancer Imaging 2005; 5:73-7.

19. Warshauer DM, Hall HL. Solitary splenic lesions. Semin Ultrasound CT MR 2006; 27:370-88.

20. Abbott RM, Levy AD, Aguilera NS, Gorospe L, Thompson WM. From the archives of the AFIP: primary vascular neoplasms of the spleen: radiologicpathologic correlation. Radiographics 2004; 24:1137-63.

21. Kamaya A, Weinstein S, Desser TS. Multiple lesions of the spleen: differential diagnosis of cystic and solid lesions. Semin Ultrasound CT MR 2006; 27:389-403.

22. Bhatia K, Sahdev A, Reznek RH. Lymphoma of the spleen. Semin Ultrasound CT MR 2007; 28:12-20.

23. Thanos L, Dailiana T, Papaioannou G, et al. Percutaneous CT-guided drainage of splenic abscess. AJR Am J Roentgenol 2002; 179:629-32.

24. Elsayes KM, Narra VR, Mukundan G, et al. MR imaging of the spleen: spectrum of abnormalities. Radiographics 2005; 25:967-82.

25. Vanhoenacker FM, De Backer Al, Op de BB, et al. Imaging of gastrointestinal and abdominal tuberculosis. Eur Radiol 2004; 14 Suppl 3:E103-15.

26. Moeller TB, Reif E. Normal findings in CT and MRI. New York: ThiemeVerlag, 2000.

27. Prassopoulos P, Daskalogiannaki M, Raissaki M, Hatjidakis A, Gourtsoyiannis N. Determination of normal splenic volume on computed tomography in relation to age, gender and body habitus. Eur Radiol 1997; 7:246-8. 


\section{SINGAPORE MEDICAL COUNCIL CATEGORY 3B CME PROGRAMME} (Code SMJ 201503B)

Question 1. Regarding imaging modalities of the spleen:

(a) Delayed phase imaging performed 3 min after contrast injection is helpful for excluding splenic lacerations in post-traumatic patients.

(b) Colour Doppler offers no additional value in splenic imaging.

(c) After intravenous contrast injection, inhomogeneous enhancement during the arterial and early portal venous phase is a sign of lymphomatous involvement of the spleen.

(d) Splenic signal intensity varies with patient age.

Question 2. The following is not a vascular tumour:
(a) Haemangioma.
(b) Littoral cell angioma.
(c) Lymphangioma.
(d) Angiosarcoma.

Question 3. The following criteria are in favour of benign cystic lesions:
(a) Intralesional solid components.
(b) Wall-thickening.
(c) Sharply circumscribed lesion.
(d) Contrast-enhancement.

Question 4. Regarding splenic infarctions:

(a) Splenic infarctions can be of either arterial or venous origin.

(b) Gas in an infarcted area is a sign of superinfection.

(c) On MR imaging, the signal intensity of infarcted areas varies depending on the age of the lesion.

(d) Chronic infarcts may decrease in size, resulting in retraction of the splenic capsule.

Question 5. Concerning significant splenic variations:

(a) Absence of free abdominal or perisplenic fluid and normal splenic enhancement are key imaging features to differentiate splenic lobules from an area of laceration.

(b) Scintigraphy with Tc-99m sulfur colloid or denatured red cells is the most sensitive technique for detecting splenules and ectopic splenic peritoneal implants.

(c) Asplenia and polysplenia are congenital syndromes associated with anomalies in viscero-atrial situs.

(d) Splenic lobules typically have irregular borders.

\footnotetext{
Doctor's particulars:

Name in full

MCR number

Specialty:

Email address

SUBMISSION INSTRUCTIONS:

(1) Log on at the SMJ website: http://www.sma.org.sg/publications/smjcurrentissue.aspx and select the appropriate set of questions. (2) Provide your name, email address and MCR number. (3) Select your answers and click "Submit".

RESULTS:

(1) Answers will be published in the SMJ May 2015 issue. (2) The MCR numbers of successful candidates will be posted online at the SMJ website by 4 May 2015. (3) Passing mark is $60 \%$. No mark will be deducted for incorrect answers. (4) The SMJ editorial office will submit the list of successful candidates to the Singapore Medical Council. (5) One CME point is awarded for successful candidates.

Deadline for submission: (March 2015 SMJ 3B CME programme): 12 noon, 27 April 2015.
} 